\title{
PENGUIn multi-instrument observations of dayside high-latitude injections during the 23 March 2007 substorm
}

\author{
M. R. Lessard, ${ }^{1}$ A. T. Weatherwax,${ }^{2}$ M. Spasojevic, ${ }^{3}$ U. S. Inan, ${ }^{3}$ A. Gerrard, ${ }^{4}$ \\ L. Lanzerotti, ${ }^{4}$ A. Ridley, ${ }^{5}$ M. J. Engebretson, ${ }^{6}$ N. J. Petit, ${ }^{7}$ R. Clauer, ${ }^{8}$ J. LaBelle, ${ }^{9}$ \\ S. B. Mende, ${ }^{10}$ H. U. Frey, ${ }^{10}$ V. A. Pilipenko, ${ }^{11}$ T. J. Rosenberg, ${ }^{12}$ and D. Detrick ${ }^{12}$
}

Received 17 June 2008; revised 14 September 2008; accepted 13 November 2008; published 29 January 2009.

[1] This paper presents ground-based observations from Antarctic stations during a substorm observed on 23 March 2007. Using fluxgate magnetometer data, supported by numerical modeling, the locations of the stations are shown to straddle the open-closed magnetic field boundary. Near these locations (on closed field lines), VLF and riometer signatures are observed to show effects of energetic particle precipitation in the morning sector (extending to the postdawn region), confirmed by observations at geosynchronous orbit. In the VLF data, both the initial injection as well as echoes are observed. The mechanism responsible for such high-latitude injections is thought to be a combination of dynamics of the injection process and drift-shell splitting. Further work will address whether similar observations can be used to infer the dynamics and/or location of the injection region.

Citation: Lessard, M. R., et al. (2009), PENGUIn multi-instrument observations of dayside high-latitude injections during the 23 March 2007 substorm, J. Geophys. Res., 114, A00C11, doi:10.1029/2008JA013507.

\section{Introduction}

[2] On 23 March 2007, the THEMIS array of satellites acquired one of its first significant observations of a substorm, shortly after becoming operational. Ground-based observations indicate that the substorm was initiated over Alaska, with an onset time of approximately 1120 UT. Various aspects of the event are discussed in other papers, including an overview of the event [Angelopoulos et al., 2008], a numerical perspective of its development (J. Raeder et al., Open GGCM simulations for the THEMIS mission,

\footnotetext{
${ }^{1}$ Space Science Center, University of New Hampshire, Durham, New Hampshire, USA.

${ }^{2}$ Department of Physics, Siena College, Loudonville, New York, USA.

${ }^{3}$ Space Telecommunications and Radioscience Laboratory, Stanford University, Palo Alto, California, USA

${ }^{4}$ New Jersey Institute of Technology, Newark, New Jersey, USA

${ }^{5}$ Center for Space Environment Modeling, University of Michigan, Ann Arbor, Michigan, USA.

${ }^{6}$ Department of Physics, Augsburg College, Minneapolis, Minnesota, USA.

${ }^{7}$ Department of Computer Science, Augsburg College, Minneapolis, Minnesota, USA.

${ }^{8}$ Virginia Institute of Technology, Blacksburg, Virginia, USA.

${ }^{9}$ Department of Physics, Dartmouth College, Hanover, New Hampshire, USA.

${ }^{10}$ Space Sciences Laboratory, University of California, Berkeley, California, USA.

${ }^{11}$ Institute of the Physics of the Earth, Moscow, Russia

${ }^{12}$ Institute for Physical Science and Technology, University of Maryland, College Park, Maryland, USA.
}

Copyright 2009 by the American Geophysical Union. 0148-0227/09/2008JA013507 submitted to Space Science Reviews, 2008), its multiple activations [Keiling et al., 2008] and ground observations of magnetic signatures [Milling et al., 2008; Russell et al., 2008]. Liu et al. [2008] present data from THEMIS and the LANL satellites, as well as model results of energetic particle injections associated with the substorm.

[3] This paper complements that of Liu et al. [2008] in that we present ground-based observations of the event from Antarctica, showing that energetic particle injections (including echoes) associated with the substorm reached the ionosphere at latitudes very near the open-closed fieldline boundary on the morning side. The evidence for the precipitation comes from VLF and riometer signatures; the specific locations of the stations is determined from the fluxgate magnetometer data, supported by numerical estimates.

[4] At geosynchronous orbit, injected particles appear with a distinct onset and an abrupt increase in count rate, followed by a relatively gradual decrease in countrate. This effect is thought to result, in principle, from dynamics of electric and magnetic fields that energize particles at substorm onset. Echoes resulting from gradient-curvature drift motion usually follow the injection, but velocity dispersion results in any subsequent peaks having decreased amplitude and a broadening of the signature with each echo. Injections have long been observed from geosynchronous orbit [Lanzerotti et al., 1967; Arnoldy and Chan, 1969; Pfitzer and Winckler, 1969; Reeves et al., 1990], while few reports exist that show in situ observations of injections at high latitudes. One exception is presented by Takahashi et al. [1997], who show AMPTE CCE observations of ion 
injections at $1300 \mathrm{MLT}$ and near $8.8 R_{E}$, associated with pseudo-substorm onsets.

[5] On the other hand, indirect observations of energetic particle precipitation have been acquired using the X-ray and far-ultraviolet instruments on Polar [Christensen et al., 2003], where an intensification associated with substorm onset was recorded at 1900 MLT and $74^{\circ}$ invariant latitude (ILAT). Additional evidence for the particle precipitation in that event was also provided by imaging riometers and photometers located at the South Pole and at Iqaluit, Nunavut. Imhof et al. [1988] also show X-ray observations of high-latitude injections, using data acquired by the S81-1 satellite. By comparing their observations with the $A E$ index, they conclude that the majority of events are not associated with substorms, however.

[6] From the ground, an increasing amount of evidence is showing that injections and subsequent echoes can be observed using VLF receivers and riometers. Abel et al. [2006] present VLF observations of substorm chorus events and drift echoes using data from Halley, Antarctica $(L=4.4$, ILAT $=\sim 62^{\circ}$ ). This paper also cites additional reports of substorm-enhanced chorus observed at many other stations, but only as poleward as $L=7.1$. In the study presented here, PENGUIn data provide a multi-instrument perspective to show evidence of injections at very high latitudes, close to the open-closed boundary.

\section{Data Presentation}

[7] The data shown here were acquired as part of the Polar Experiment Network for Geospace Upper-atmosphere INvestigations (PENGUIn) project, which supports observatories across Antarctica, instrumented to measure various ionospheric parameters with magnetometers, riometers, VLF and HF receivers, optical and other related instruments [Rosenberg and Doolittle, 1994; Engebretson et al., 1997]. More information, including data, are available at www. antarcticspacescience.org.

[8] The locations of the observatories are presented in Figure 1. The chain of stations highlighted in red are those relevant to this study and fall along a magnetic meridian delineated by the geomagnetic and geographic South Poles. The sites P2, P1 and P5 are Automated Geophysical Observatories, powered with wind generators and solar panels. Data from each of these stations is transmitted to the US via Iridium modems continuously throughout the year. Stations along this meridian, including the geographic South Pole, range from $\sim 70^{\circ}$ to $\sim 87^{\circ}$ ILAT, with additional data available from Halley Bay. For reference, sites along this meridian are approximately conjugate to points in the northern hemisphere along the east coast of Canada [Dudeney et al., 1997]. Note that the P2, P1 and P5 designations stand for "PENGUIn" and are not related to similar designations in the THEMIS satellite.

\subsection{Overview}

[9] In the conventional view of substorm progression, bright auroral features expand rapidly poleward in the midnight sector and reach latitudes well above the typical location of the nightside oval, often accompanied by westward and eastward movements. In fact, the maximum poleward extent of auroral precipitation effects can easily

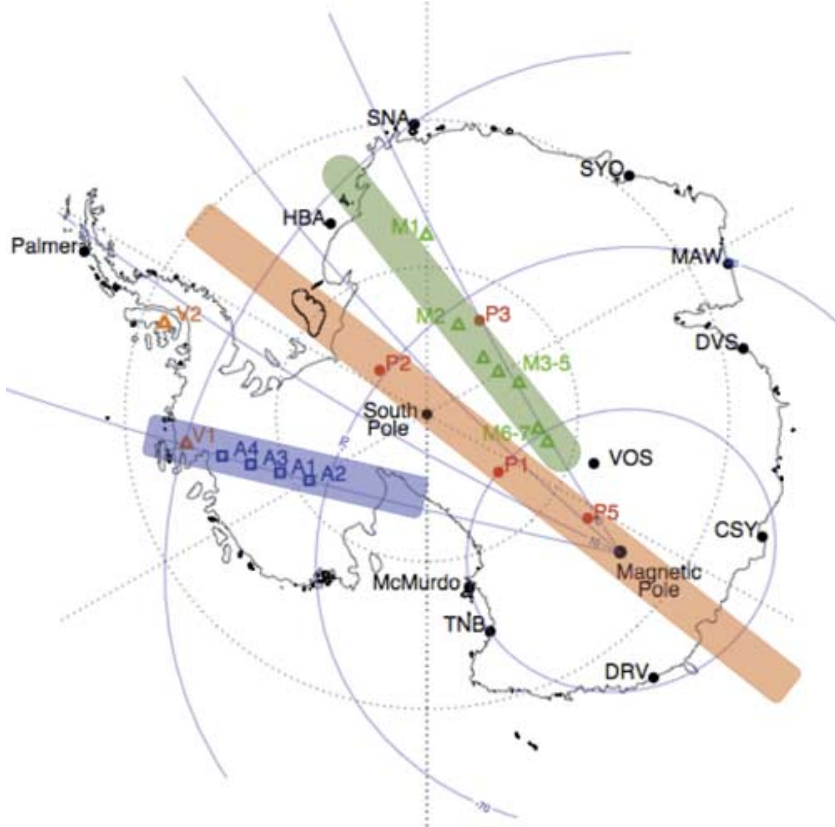

Figure 1. Map showing observatory locations in Antarctica. The stations highlighted in red are most relevant to this study and lie along a meridian spanning $\sim 70^{\circ}$ to $\sim 87^{\circ}$ ILAT.

reach latitudes of $75^{\circ}$ magnetic latitude, and can sometimes extend beyond $80^{\circ}$. See Gussenhoven [1982]; Craven and Frank [1991]; Weatherwax et al. [1997] and Mende et al. [1999] and references therein for morphological descriptions of high-latitude substorms.

[10] A similarity in conditions shared by many substorms that reach high latitudes is the occurrence of relatively high solar wind speeds. Weatherwax et al. [1997], using riometer data, found that of 24 substorm events seen at high latitude, all occurred when $V_{s w}>700 \mathrm{~km} / \mathrm{s}$. A necessity for high solar wind speed conditions in order to see substorms at high latitudes was also reported by Sergeev et al. [1979], based on observations made at Vostok station, Antarctica. In this particular example, however, the solar wind speed (not shown) was only the order of $\sim 320 \mathrm{~km} / \mathrm{s}$, which raises questions regarding what controls the poleward development of substorms.

[11] Figure 2, to be discussed in more detail below, provides an overview of the activity observed at the South Pole from 0900 to 1500 UT on 23 March. The top three plots show the $\mathrm{H}, \mathrm{D}$ and $\mathrm{Z}$ axes of the fluxgate magnetometer. The fourth plot shows riometer absorptions. The bottom two plots show $0.5-1$ and $1-2 \mathrm{kHz}$ VLF data, respectively. In contrast with lower-latitude magnetometer data, which shows a clear onset near 1110 UT [Russell et al., 2008], no clear onset is observed at the South Pole. Rather, the fluxgate and induction coil magnetometers both measure significant activity throughout the day (not shown), long before and after the occurrence of the substorm.

[12] Undoubtedly, the lack of a distinct electrojet signature at onset is due to the relatively high magnetic latitude of the Pole (-74.0 ILAT), as well as the fact that it is located near 7.5 MLT at the time of the onset. On the other hand, we 

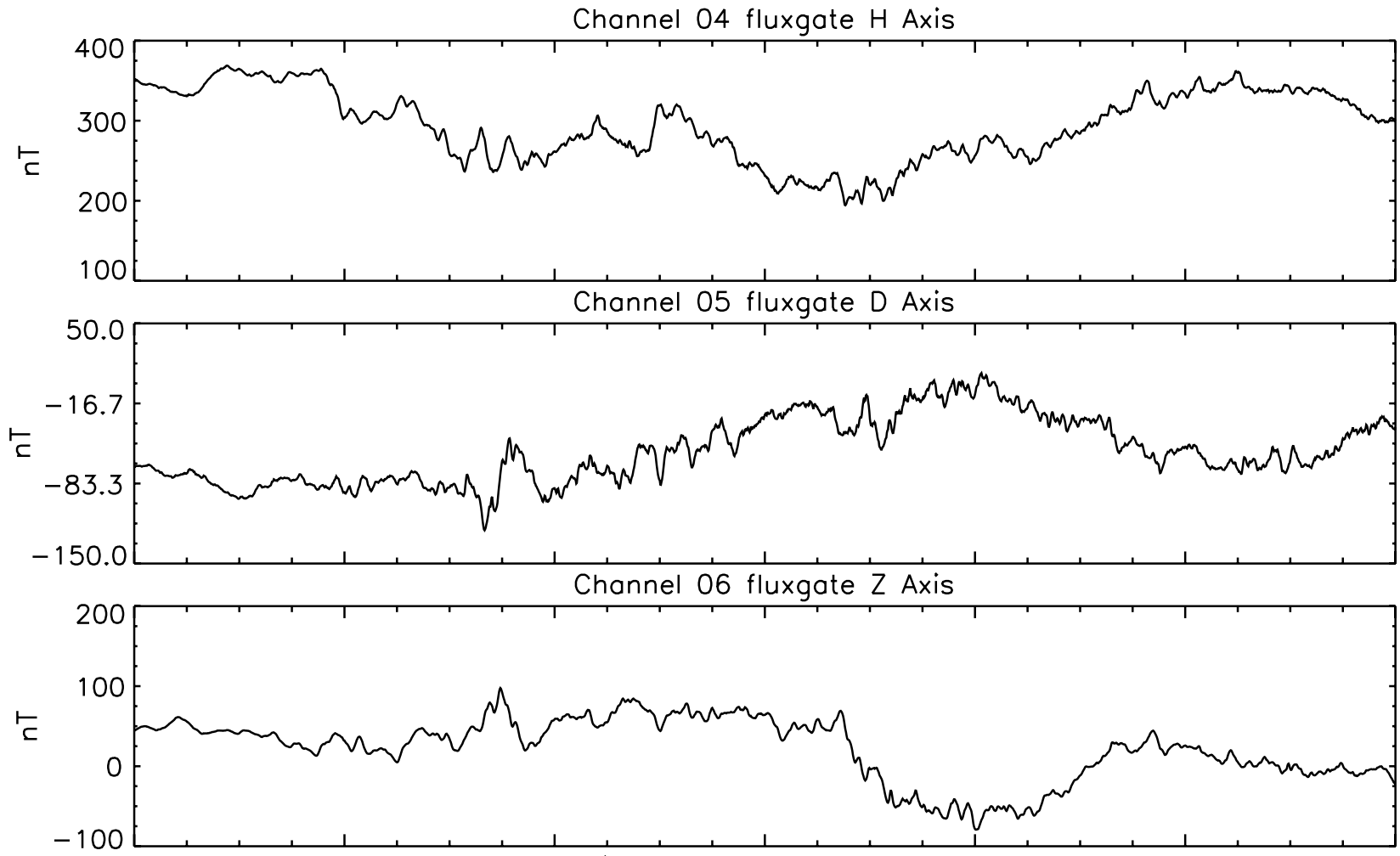

Channel 09 Riometer 38Mhz QDC=PSpa07_03.qdc
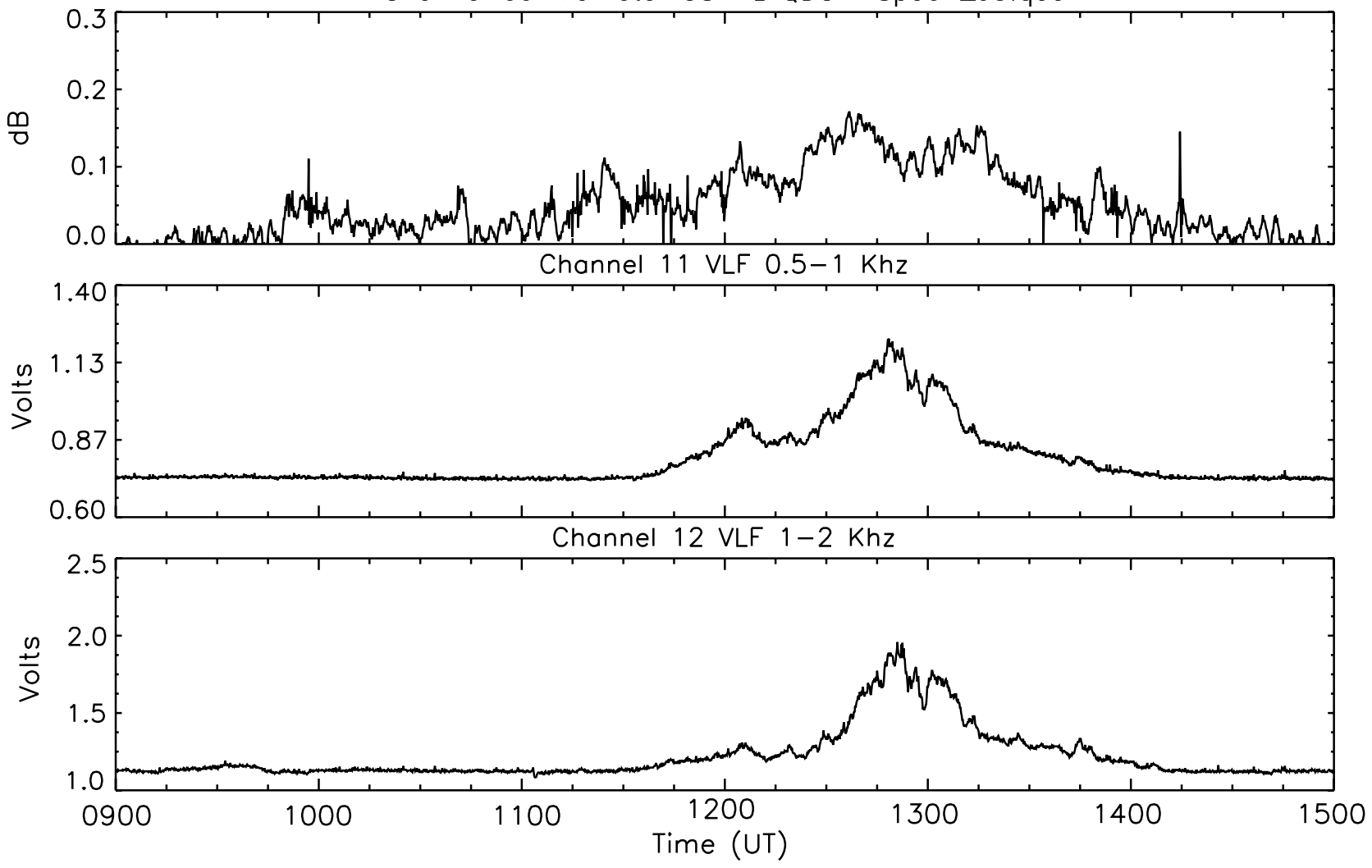

South Pole DAS, 23 March 2007, day 82

Figure 2. Data from the South Pole from 23 March 2007 from 0900 to 1500 UT. From top to bottom are plotted 3 axes of fluxgate magnetometer data, the north-south component of the induction coil magnetometer, riometer absorptions, and VLF signals (bottom two plots). 

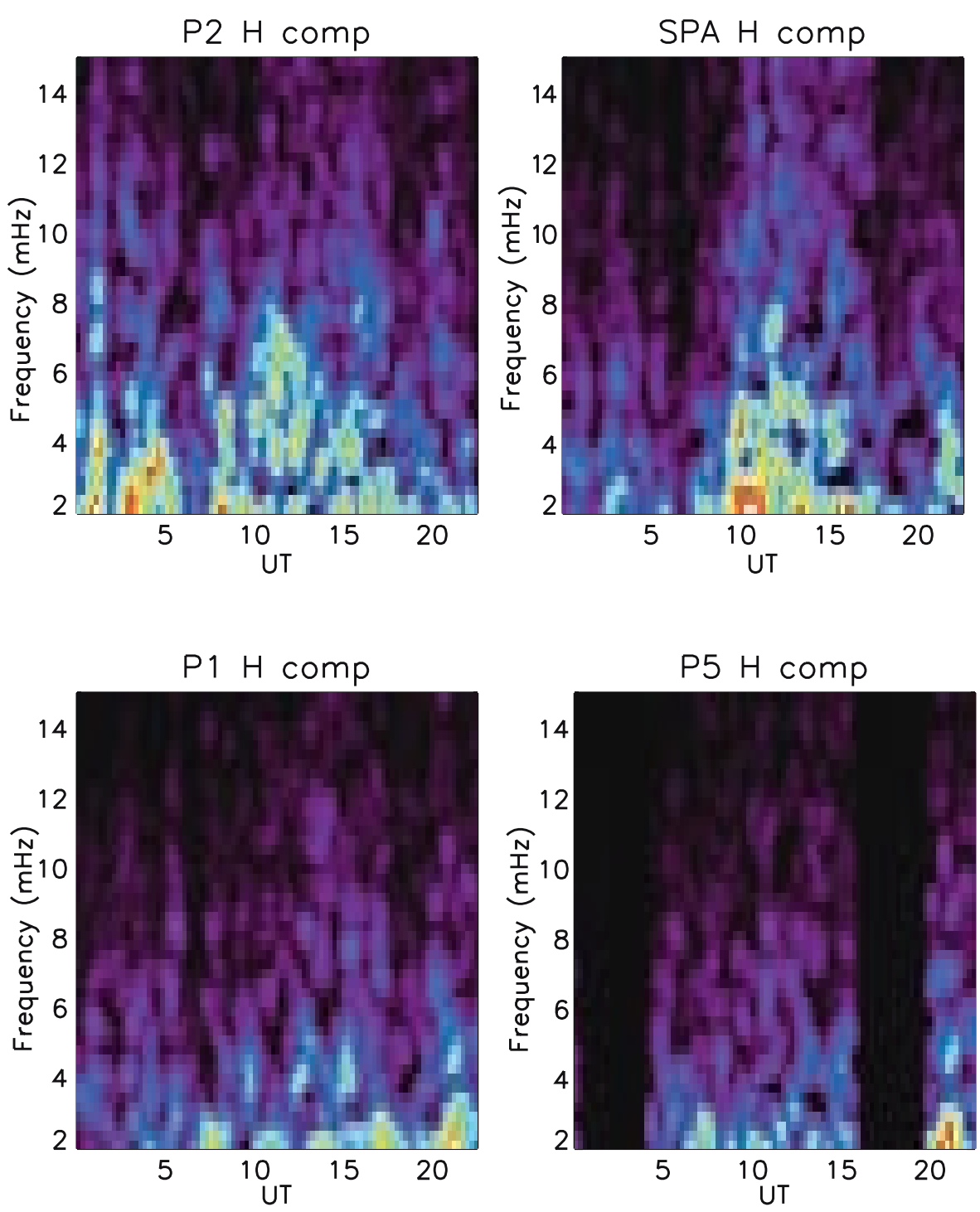

Figure 3. H-component fluxgate magnetometer spectra for various sites, located at $-69.9(\mathrm{P} 2),-74.0$ (SPA), -80.1 (P1), and -86.6 ILAT (P5). The lack of spectral power near a few mHz (e.g., at P5) implies the station is on open field lines.

show below that energetic electron injections are observed in the vicinity of the South Pole as well as at nearby remote sites. Again, aspects of substorm development at high latitudes remain unclear.

\subsection{Open-Closed Field Line Boundary}

[13] Lanzerotti et al. [1999] used PENGUIn magnetometer data, together with data from overflights of a polarorbiting satellite to determine the location of the openclosed boundary by examining its spectral properties, noting the occurrence or lack of occurrence in a narrow band of spectral power in the range of a few $\mathrm{mHz}$. Specifically, they showed that there is a demarcation in the occurrence of these emissions in the magnetosphere between magnetic latitudes of $\sim 75^{\circ}$ and $\sim 80^{\circ}$, consistent with the findings of Waters et al. [1995] and of Mathie et al. [1999], who analyzed data from latitudinal arrays of magnetometers at geomagnetic latitudes $\leq 75^{\circ}$. Since the narrow band emission is typically seen simultaneously at more than one station, the authors conclude is unlikely that it arises from a single magnetopause feature such as the cusp (including particle precipitation into the cusp to produce waves). Instead, they conclude that the spectra are providing evidence of a plasma density feature in the magnetosphere, the magnetopause itself. That is, they conclude that the spectra probably provide evidence of Alfvén waves that exist on closed flux tubes at the locations of the various stations and, therefore, that an array of high geomagnetic latitude stations can be used to determine the location of the Earth's magnetopause on the dayside of the magnetosphere.

[14] Following Lanzerotti et al. [1999], magnetometer spectra were used to determine the approximate location of open-closed boundary during the 23 March substorm and the period that followed it. Spectra were calculated using data from stations located at $-69.9^{\circ}(\mathrm{P} 2),-74.0^{\circ}$ (SPA), $-80.1^{\circ}(\mathrm{P} 1)$ and $-86.6^{\circ}(\mathrm{P} 5)$. Figure 3 shows the results. From 0000-0800 UT, field lines at P2 are closed while those at the South Pole, P1 and P5 are open. Actually, field 

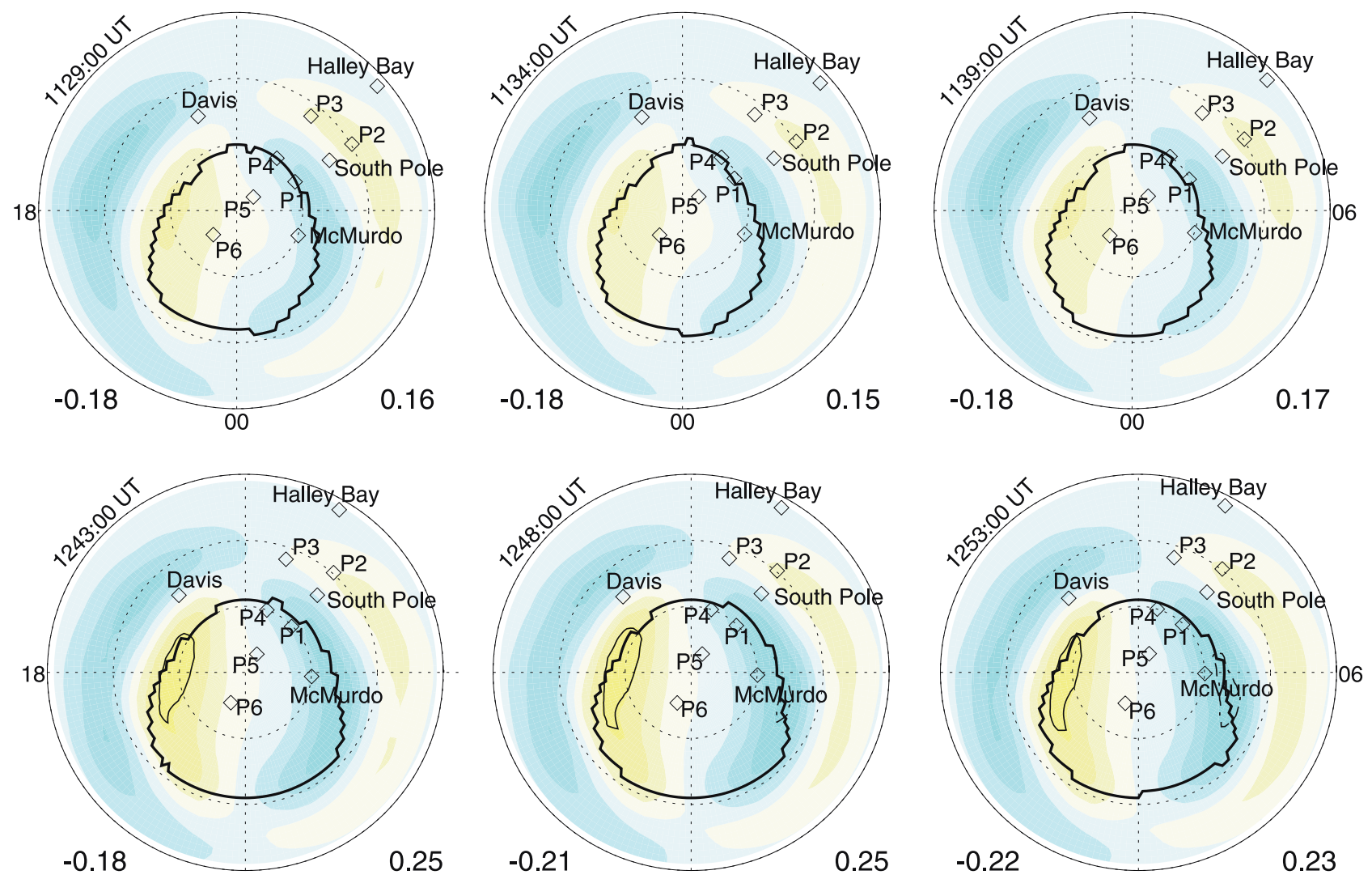

Figure 4. BATSRUS estimate of the open-closed boundary. The top row shows estimates for a duration of 10 minutes, beginning at 1129 UT (substorm onset). The bottom row also shows 10 minutes of data, but beginning near 1243 UT, the approximate time that substorm injections were observed at the Pole (discussed below).

lines at P2 are closed and those at P5 are open throughout the entire day. From 0800-0900 UT, the open-closed boundary moves over the Pole toward P1, placing the South Pole in a region of closed field lines. From 0900 UT and throughout the remainder of the day, the boundary remains between the Pole and P1, but must be much closer to P1.

[15] A numerical estimate of the location of the openclosed boundary was also made using the Block Adaptive Tree Solar-wind Roe-type Upwind Scheme (BATSRUS) MHD code [Powell et al., 1999], which describes solarwind magnetosphere interactions. The BATSRUS code has a resolution of $1 / 8 R_{E}$ in the near-Earth region and $1 / 4 R_{E}$ grid throughout the dayside and nightside of the magnetosphere. Results are presented in Figure 4.

[16] The top row of Figure 4 shows the estimated location of the open-closed boundary near the time of substorm onset, suggesting that P1 must be on closed field lines (with the boundary in between P1 and P5), although the boundary remains very close to $\mathrm{P} 1$ throughout the interval. This is consistent with the conclusion based on the magnetometer data, which indicate that the open-closed boundary was between P1 and the Pole, but very near P1, as well as with the T96 model estimate, as shown in Figure 6.

[17] The bottom row of Figure 4 shows the estimate at a time corresponding to approximately the drift echo time following onset (i.e., near $1240 \mathrm{UT}$ ). In this case, both the model results and the magnetometer analysis conclude that the boundary must be very near P1, perhaps between P1 and the Pole. This is significant because, as shown in the next section, it places the location ground-based observations of substorm injections very near the boundary.

\subsection{High-Latitude Substorm Injections}

[18] Chorus is an intense whistler-mode wave that permeates the low-density region between the plasmapause and the magnetopause and can be observed on the ground over a range of latitudes [e.g., Sazhin and Hayakawa, 1992, and references therein]. Chorus is believed to be generated through the electron cyclotron instability by anisotropic $\left(T_{\perp}>T_{\|}\right)$distributions of energetic electrons in the range of 5 to $150 \mathrm{keV}$ [Tsurutani and Smith, 1974; Thorne et al., 1977]. These unstable distributions can result from substorm particle injections, and correspondingly, chorus is predominantly observed across the morning-side of the Earth in association with the eastward drifting electrons [e.g., Burtis and Helliwell, 1976]. Indeed, in the middle magnetosphere ( $L \simeq 4$ to 8 ) the onset of wave generation has been well associated with substorms. In particular, Tsurutani and Smith [1974] first showed that the distribution of chorus as a function of $L$-shell and MLT in the postmidnight sector was similar to the enhanced substorminjected electrons with energies $>40 \mathrm{keV}$.

[19] The general morphology of chorus observed in the outer dayside magnetosphere is quite different from that at lower L values. Tsurutani and Smith [1977] reported that the emissions are detected on the dayside, at $8 \leq$ MLT $\leq 16$, and 


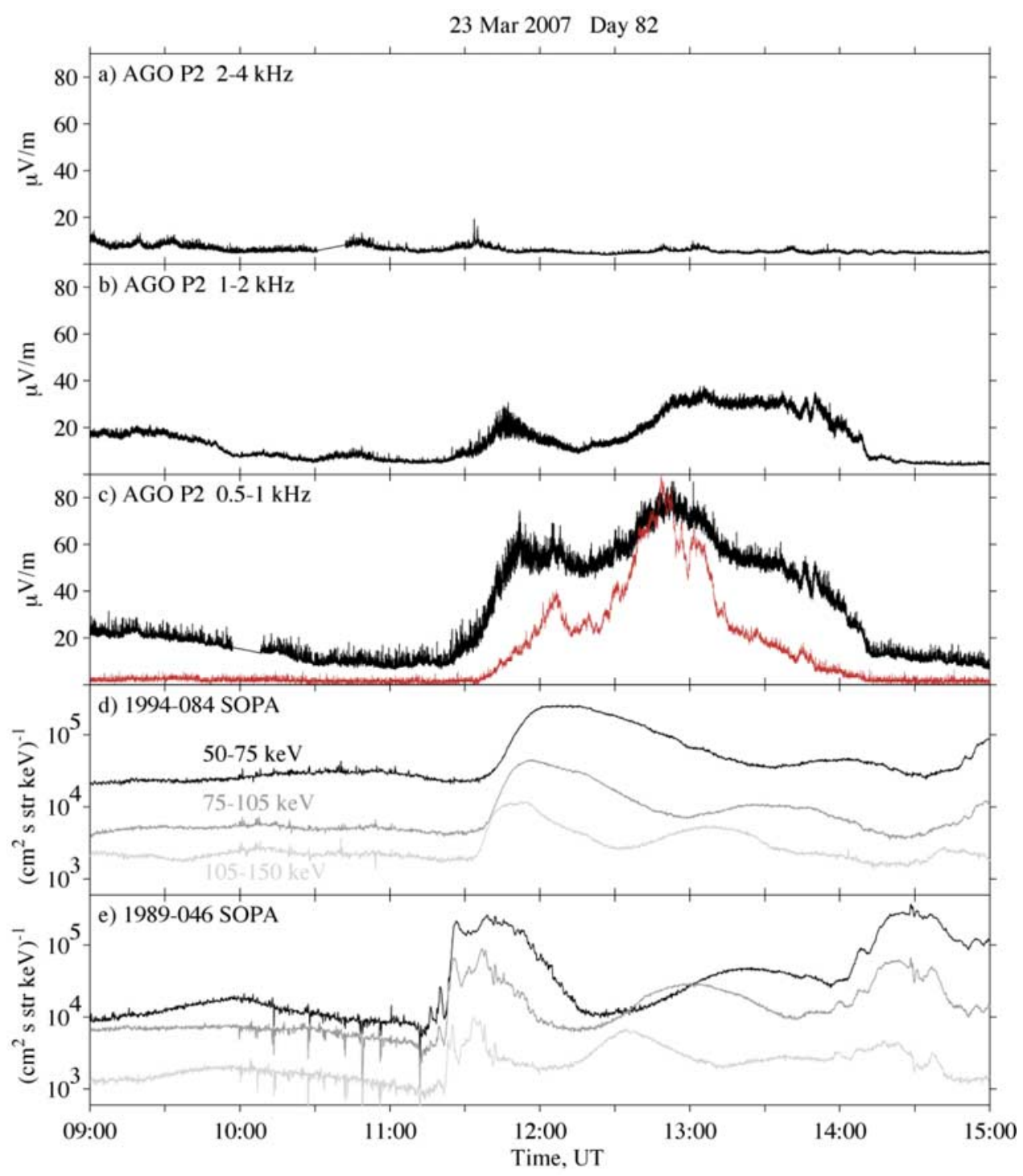

Figure 5. Chorus observations from AGO P2 along with energetic electron measurements from the SOPA instrument on two geosynchronous spacecraft, 1989-046 and 1994-084. The red trace shows chorus observed at the South Pole.

within few $\mathrm{R}_{\mathrm{E}}$ of the magnetopause. While chorus observed at lower $L$ on the dawn-side is generated at the geomagnetic equator, chorus on the dayside appears to be generated at latitudes greater than 15 degrees, possibly in so called "minimum-B pockets" [Tsurutani and Smith, 1977; Meredith et al., 2001]. These are regions of relatively low magnetic field in the off-equatorial region that result from solar wind compression of the dayside magnetopause. Owing to the off-equatorial generation, Tsurutani and Smith [1977] dubbed these emissions high-latitude chorus, and the same emission is referred to as polar chorus when observed on the ground as a result of its measurement at high invariant latitude.

[20] Tsurutani and Smith [1977] found no statistical correlation between the high-latitude chorus and substorms as measured by the AE index. Although some of the events did appear in the aftermath of substorms, many of the chorus observations occurred during periods of extreme geomagnetic quiet. They did, however, note that the waves could be related to substorms occurring at invariant latitudes higher than those measured by the AE index [e.g., Kamide and Akasofu, 1974].

[21] Figure 5 shows chorus observations from AGO P2 and the South Pole along with energetic electron measurements from the SOPA instrument on two geosynchronous spacecraft, 1989-046 and 1994-084. Only the lowest three energy channels are displayed since these energies are most likely associated with chorus generation. Black traces in Figures $5 \mathrm{a}-5 \mathrm{c}$ show chorus observed at P2; the red trace shows chorus observed at the Pole. This plot shows that chorus signatures (Figures $5 \mathrm{a}-5 \mathrm{c}$ ) coincide closely (in time) with particle injections observed at geosynchronous orbit. It also shows that the observation at the South Pole lagged behind that of P2 by approximately 10 minutes (discussed below).

[22] Figure 6 indicates the locations of ground station and spacecraft mapped along magnetic field lines to the GSM equatorial plane at the approximate time of the substorm 


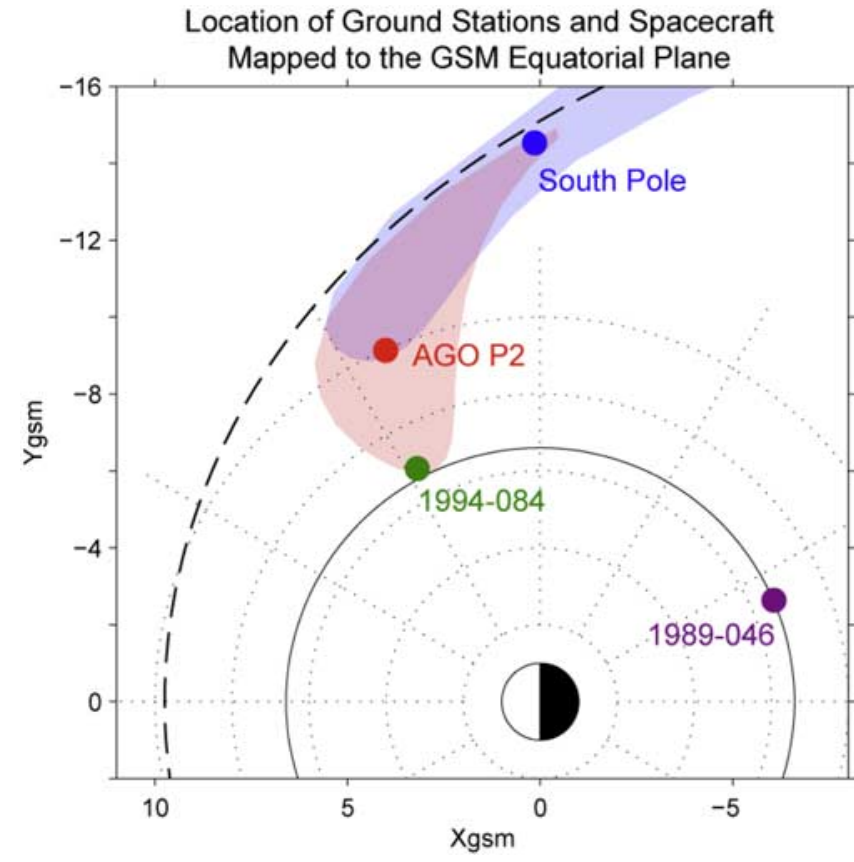

Figure 6. The locations of ground station and spacecraft mapped along magnetic field lines to the GSM equatorial plane (based on the T96 model) at the approximate time of the substorm onset.

onset using the T96 magnetic field model [Tsyganenko and Stern, 1996] and the prevailing solar wind conditions. At 11:23 UT, 1989-046, located in the postmidnight sector, observed a typical signature of a substorm electron injection (Figure 5e). About 15 minutes later, 1994-084, located near 8 MLT, begins to observe the drifting energetic electrons (Figure 5d). The differential drift can be clearly seen with the highest-energy electrons arriving first. AGO P2 observes the onset of chorus (Figures 5a-5c) about five to seven minutes after the initial injection is observed by 1989-046. Although AGO P2 and 1994-084 are located near the same magnetic longitude, P2 observes chorus before the electrons have drifted to 1994-084. ELF/VLF broadband snapshot recordings, Figure 7, verify that the emissions observed in the narrowband channels are indeed chorus with the typical spectrum of overlapping, discrete rising tones being clearly observed.

[23] The onset of chorus at P2 before the observations of actual injections at $1994-084$ is interesting. First, recall that the energy channels displayed in Figure 5 are those most likely associated with chorus generation and thus should represent information about the same particle population. Then, since the drift speed for particles of any given energy is faster at higher L shells, one might expect particles of comparable energies to be observed at higher latitudes (i.e., at 1994-084) first. Why this does not appear to be the case in these data likely is a result of two effects. First, the locations of the platforms, as estimated by the T96 should not be so strictly interpreted. Secondly, ground observations of chorus provided a spatially integrated measure of chorus rather than a point measurement. The "viewing area" of the ground observations, is shown in Figure 6 and described below.

[24] Chorus observations at South Pole show a similar pattern to the activity observed at P2. The red trace in Figure 5 shows the onset of chorus after a delay of roughly 10 minutes following the onset at P2. The receiver at P2 recorded a double-peak in chorus (near 1150 and 1205 UT) coinciding with the first injection, while only the second peak (associated with the same injection) is observed at the Pole.

[25] A subsequent peak in the chorus activity is observed at P2 and at South Pole near 1250 UT. This peak appears to be related to the drift echo of the energetic electrons that is seen by both spacecraft as the electrons drift a full orbit around the Earth. This is similar to a recent report of substorm chorus events at lower latitudes. Abel et al. [2006] showed that at an $L=4$ ground station about $25 \%$ of substorm chorus events were associated with the drift echo rather than the initial substorm injection. A third peak in the energetic electron fluxes can be seen at about

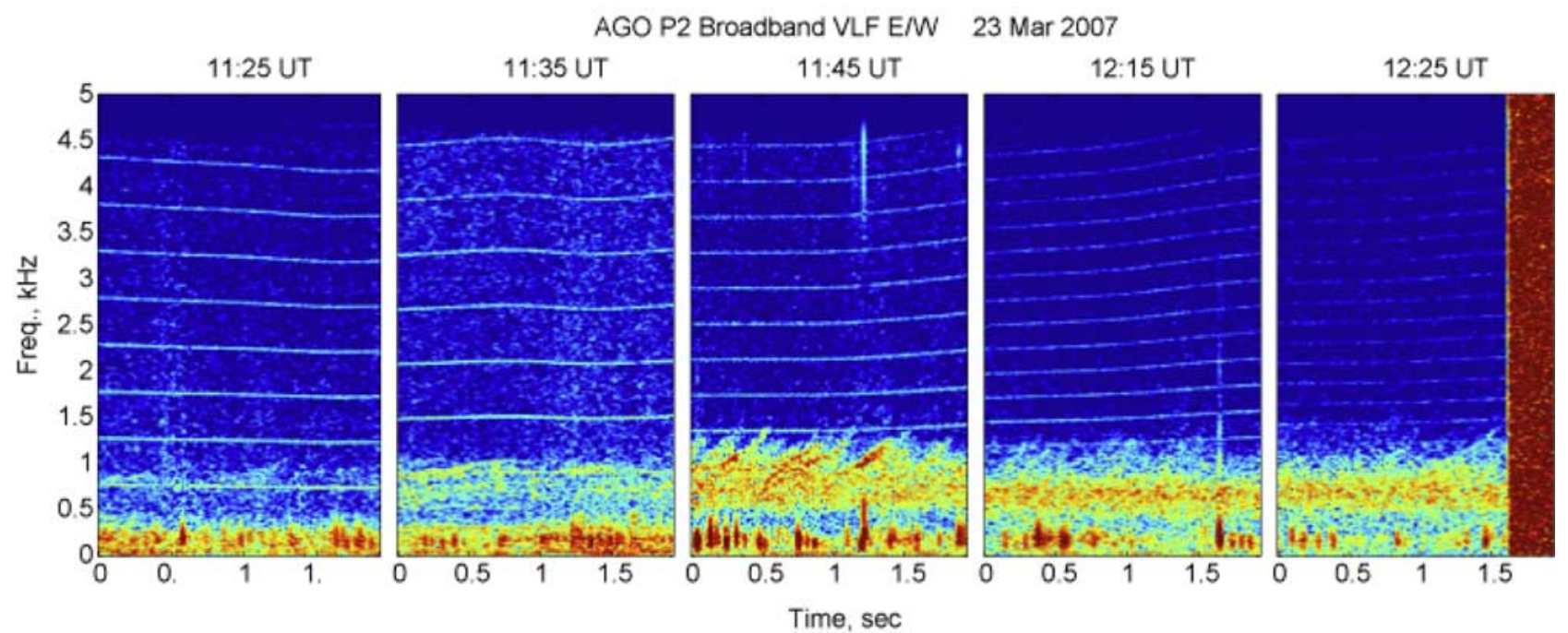

Figure 7. ELF/VLF broadband snapshot recordings from P2. 
14:30 UT, but there is no corresponding chorus onset at P2 or South Pole. Chorus is not observed for the remainder of the day.

[26] The shaded areas in Figure 6 are the estimated ELF/ VLF viewing areas of the station. The viewing area is $500 \mathrm{~km}$ circle around the station that is then mapped out to the equator. Clearly, the South Pole maps very close to the magnetopause and significantly west of AGO P2, thus it is not unexpected that SP and P2 see slightly different wave signatures. We note, also, that the P1 location was estimated to be on open field lines by the model, consistent with the conclusions extracted from the data, above.

\subsubsection{Riometer Observations}

[27] Returning to Figure 2, weak (but clear) riometer absorptions can be seen in the fourth plot. Recently, riometer data have increasingly been associated with energetic particle injections at substorm onset, typically having excellent correlations with satellite observations at auroral latitudes [Spanswick et al., 2007; Liang et al., 2007; Liu et al., 2007]. Riometer absorptions represent changes in ionospheric conductivity associated with particle precipitation and complement VLF observations in the sense that absorptions are not ducted (as VLF waves are) and therefore show that the precipitation is relatively close to a station (within a few hundred $\mathrm{km}$ ).

[28] In Figure 2, the absorption is weak and gradual, perhaps indicating that the particle precipitation is nearly out of the instrument field-of-view. Still, with several indicators showing that the South Pole is situated quite close to the open-closed boundary, these data consistently indicate that particle injections are reaching the ground at very high latitudes.

\section{Summary and Discussion}

[29] In conjunction with the onset of the 23 March 2007 substorm, riometers and VLF receivers at high-latitude ground stations AGO P2 and South Pole station observe clear signatures of energetic particle precipitation, coinciding with substorm-injected electrons observed by two geosynchronous spacecraft. The ground stations were located in the postdawn local time sector on field lines that map to greater than $10 R_{\mathrm{E}}$ (very close to the open-closed boundary), as determined numerically and through analysis of the fluxgate magnetometer data. A secondary peak in the chorus activity about an hour later appears to be related to the electron drift echo. A third peak in the energetic electron flux at 1430 UT at geosynchronous does not have a corresponding chorus onset at P2 or South Pole. By this time, the stations had rotated to near the noon meridian, and although this is still within the typical diurnal chorus window (in fact at these latitudes, chorus occurs most frequently near local noon), no chorus was observed.

[30] From a theoretical standpoint, dynamics of substorm injections have primarily concentrated on replicating signatures of injections observed at geosynchronous orbit. In the model of X. Li and collaborators, the earthward dipolarization of the magnetic field results in an inductive electric field from Faraday's Law, with the perturbation starting first near midnight and then spreading in azimuth in both directions. The dawn-dusk component of the electric field, which also propagates earthward, can cause the charged particles to move earthward, following the motion of the ExB drift. In this process, the particles will be energized through betatron acceleration [Li et al., 2003; Sarris and Li, 2005]. On the other hand, the inclusion of particles only with pitch angles of $90^{\circ}$ means that this model does not address drift-shell splitting effects.

[31] Similar work has been carried out by Birn et al. [1998] and Birn et al. [1997], using a 3-D MHD code, and again targeting geosynchronous observations. More recently, analytical orbit solutions for nonrelativistic particles interacting with a cylindrical wave pulse of a trapezoidal radial field profile and a finite azimuthal extent were described by Zaharia et al. [2000]. Subsequently, Zaharia et al. [2004] also have incorporated a time-dependent, nondipolar model, but still concentrate on observations at geosynchronous orbit (and addressing only pitch angles of $90^{\circ}$ ). Finally, a different approach, where electrons are accelerated via a combination of whistler-mode waves and compressional ULF turbulence at $2-15 \mathrm{mHz}$ near the geosynchronous orbit, is presented by $\mathrm{Li}$ et al. [2005a] and $\mathrm{Li}$ et al. [2005b]. Note that the works of Birn et al. address all pitch angles, and in this respect are more general than the works of $\mathrm{Li}$ and Zaharia.

[32] This complexity of particle injections is clear in the results of Takahashi et al. [1997], who show ion injections observed by AMPTE CCE near its apogee of $8.8 R_{E}$ and near 13 MLT, following a pseudo-onset, and interpret the data in terms of drift-shell splitting. Using a Tsyganenko 89c model, these authors calculate drift orbits and infer that the injection inner boundary must have been located at $\sim 9 R_{E}$; essentially, they were able to "invert" their data to place constraints on the location of the injection boundary. Similar work had been carried out by Sibeck et al. [1987], who had also used AMPTE CCE data and showed that particles with $90^{\circ}$ pitch angles drifting around to the dayside can reach near the openclosed boundary, as we report here. Clearly, while the various models of injections and (separately) of drift shell splitting apparently provide important information, improvements in these models could perhaps combine these effects for a proper treatment of the injection process.

[33] The results presented in this paper provide the first ground-based observations of high-latitude injections in association with substorm onset (that perhaps form the ground-based counterpart to the observations of Takahashi et al. [1997] and Sibeck et al. [1987]). The injections were observed using VLF and riometer data, but confirmed with observations from geosynchronous orbit. Presumably, the mechanism that delivers these particles to high latitudes is a combination of the initial substorm injection (including the location of the injection mechanism) and drift-shell splitting. These results suggest that future theoretical efforts should include both the injection process and drift shell splitting.

[34] Finally, we note that the injections play an additional role, that of perturbing ionospheric conductivity. There is no doubt that this process causes enhancements in ionospheric conductivity (although the spatial and temporal aspects as well as the extent of the enhancement are unknown at this point). Whether this precipitation can affect the poleward evolution of substorms (e.g., through ionospheric feedback) is not known. An important step in answering these ques- 
tions will be to further characterize ionospheric signatures of injections at high (as well as at auroral) latitudes.

[35] Acknowledgments. We gratefully acknowledge the excellent field work of Will Rachelson (Cal Berkeley), Jeff Chang (Stanford), and Dan Detrick (University of Maryland), which has resulted in the successful operation of the AGO observatories. Research at the University of New Hampshire was supported by NSF grants ANT-0636874 and ANT0538474. Research at Augsburg College was supported by NSF grants ANT-0636623 and ANT-0538379, at Siena College by ANT-0636790 and ANT-0638587, at the New Jersey Institute of Technology by ANT0341046, at the University of Maryland by ANT-0636790 (to Siena College), at the University of Michigan by ANT-0636685, at Virginia Polytechnic Institute and State University by ANT-0636691, at Dartmouth College by ANT-0636798, at Stanford University by ANT-0636927, and at the University of California by ANT-0636978.

[36] Wolfgang Baumjohann thanks the reviewers for their assistance in evaluating this paper.

\section{References}

Abel, G. A., M. P. Freeman, A. J. Smith, and G. D. Reeves (2006), Association of substorm chorus events with drift echoes, J. Geophys. Res. 111, A11220, doi:10.1029/2006JA011860.

Angelopoulos, V., et al. (2008), First results from the THEMIS mission, Space Sci. Rev., in press.

Arnoldy, R. L., and K. W. Chan (1969), Particle substorms observed at geostationary orbit, J. Geophys. Res., 74, 5019-5028.

Birn, J., M. F. Thomsen, J. E. Borovsky, G. D. Reeves, D. J. McComas, R. D. Belian, and M. Hesse (1997), Substorm ion injections: Geosynchronous observations and test particle orbits in three-dimensional dynamic MHD fields, J. Geophys. Res., 102, 2325-2342.

Birn, J., M. F. Thomsen, J. E. Borovsky, G. D. Reeves, D. J. McComas, R. D. Belian, and M. Hesse (1998), Substorm electron injections: Geosynchronous observations and test particle simulations, J. Geophys. Res., 103, 9235-9248.

Burtis, W. J., and R. A. Helliwell (1976), Magnetospheric chorus: Occurrence patterns and normalized frequency, Planet. Space Sci., 24 1007-1007.

Christensen, T., N. Østgaard, T. J. Rosenberg, D. L. Detrick, G. A. Germany, and P. Stauning (2003), Conjugate high-intensity energetic electron precipitation at high latitude, Ann. Geophys., 21, 1443-1455.

Craven, J. D., and L. A. Frank (1991), Diagnosis of auroral dynamics using global aurora imaging with emphasis on large-scale evolutions, in Auroral Physics, edited by C.-I. Meng, M. J. Rycroft, and L. A. Frank, pp. 273-297, Cambridge Univ. Press, Cambridge, U. K.

Dudeney, J. R., R. B. Horne, M. J. Jarvis, R. I. Kressman, A. S. Rodger, and A. J. Smith (1997), British Antarctic Surveys ground-based activities complementary to satellite missions such as Cluster, in Satellite-Ground Based Coordination Sourcebook, edited by M. Lockwood, M. N. Wild, and H. J. Opgenoorth, Eur. Space Agency Spec. Publ., ESA-SP 1198 , $101-109$.

Engebretson, M. J., et al. (1997), The United States automatic geophysica observatory (AGO) program in Antarctica, in Satellite-Ground Based Coordination Sourcebook, edited by M. Lockwood, M. N. Wild, and H. J. Opgenoorth, Eur. Space Agency Spec. Publ., ESA-SP 1198, 65-99.

Gussenhoven, M. S. (1982), Extremely high latitude auroras, J. Geophys Res., 87, 2401-2412.

Imhof, W. L., H. D. Voss, D. W. Datlowe, and J. Mobilia (1988), Isolated electron precipitation regions at high latitudes, J. Geophys. Res., 93, 2649-2660

Kamide, Y., and S.-I. Akasofu (1974), Latitudinal cross section of the auroral electrojet and its relation to the interplanetary magnetic field polarity, J. Geophys. Res., 79, 3755-3771.

Keiling, A., et al. (2008), Multiple intensifications inside the auroral bulge and their association with plasma sheet activities, J. Geophys. Res., 113 A12216, doi:10.1029/2008JA013383.

Lanzerotti, L. J., C. S. Roberts, and W. L. Brown (1967), Temporal variations in the electron flux at synchronous altitudes, J. Geophys. Res., 72, 5893-5902.

Lanzerotti, L. J., A. Shono, H. Fukunishi, and C. G. Maclennan (1999), Long-period hydromagnetic waves at very high geomagnetic latitudes, J. Geophys. Res., 104, 28,423-28,435.

Li, L., J. Cao, and G. Zhou (2005a), Combined acceleration of electrons by whistler-mode and compressional ULF turbulences near the geosynchronous orbit, J. Geophys. Res., 110, A03203, doi:10.1029/2004JA010628.

Li, L.-Y., J.-B. Cao, G.-C. Zhou, and Z.-X. Liu (2005b), Electron acceleration by compressional ULF turbulence in the geomagnetosphere, Chin. Astron. Astrophys., 29, 235-242.
Li, X., T. E. Sarris, D. N. Baker, W. K. Peterson, and H. J. Singer (2003), Simulation of energetic particle injections associated with a substorm on August 27, 2001, Geophys. Res. Lett., 30(1), 1004, doi:10.1029/ 2002 GL015967.

Liang, J., W. W. Liu, E. Spanswick, and E. F. Donovan (2007), Azimuthal structures of substorm electron injection and their signatures in riometer observations, J. Geophys. Res., 112, A09209, doi:10.1029/2007JA012354.

Liu, W., X. Li, T. E. Sarris, C. Cully, R. E. Ergun, V. Angelopoulos, D. E. Larson, A. Keiling, K. H. Glassmeier, and U. H. Auster (2008), Observation and modeling of the injection observed by THEMIS and LANL satellites during the 23 March 2007 substorm event, J. Geophys. Res. doi:10.1029/2008JA013498, in press.

Liu, W. W., J. Liang, E. Spanswick, and E. F. Donovan (2007), Remotesensing magnetospheric dynamics with riometers: Observation and theory, J. Geophys. Res., 112, A05214, doi:10.1029/2006JA012115.

Mathie, R. A., F. W. Menk, I. R. Mann, and D. Orr (1999), Discrete field line resonances and the Alfvén continuum in the outer magnetosphere, Geophys. Res. Lett., 26, 659-662.

Mende, S. B., H. U. Frey, S. P. Geller, and J. H. Doolittle (1999), Multistation observations of auroras: Polar cap substorms, J. Geophys. Res., $104,2333-2342$.

Meredith, N. P., R. B. Horne, and R. R. Anderson (2001), Substorm dependence of chorus amplitudes: Implications for the acceleration of electrons to relativistic energies, J. Geophys. Res., 106, 13,165-13,178.

Milling, D. K., I. J. Rae, I. R. Mann, K. R. Murphy, A. Kale, C. T. Russell, V. Angelopoulos, and S. Mende (2008), Ionospheric localisation and expansion of long-period Pi1 pulsations at substorm onset, Geophys. Res. Lett., 35, L17S20, doi:10.1029/2008GL033672.

Pfitzer, K. A., and J. R. Winckler (1969), Intensity correlations and substorm electron drift effects in the outer radiation belt measured with the OGO 3 and ATS 1 satellites, J. Geophys. Res., 74, 5005-5018.

Powell, K. G., P. L. Roe, T. J. Linde, T. I. Gombosi, and D. L. de Zeeuw (1999), A solution-adaptive upwind scheme for ideal magnetohydrodynamics, J. Comput. Phys., 154, 284-309.

Reeves, G. D., T. A. Fritz, T. E. Cayton, and R. D. Belian (1990), Multisatellite measurements of the substorm injection region, Geophys. Res. Lett., 17, 2015-2018.

Rosenberg, T. J., and J. H. Doolittle (1994), Studying the polar ionosphere and magnetosphere with Automatic Geophysical Observatories: The United States program in Antarctica, Antarct. J. U. S., 29(5), 347349

Russell, C. T., P. J. Chi, D. J. Dearborn, Y. S. Ge, B. Kuo-Tiong, J. D. Means, D. R. Pierce, K. M. Rowe, and R. Snare (2008), THEMIS ground-based magnetometers, Space Sci. Rev., in press.

Sarris, T., and X. Li (2005), Evolution of the dispersionless injection boundary associated with substorms, Ann. Geophys., 23, 877-884.

Sazhin, S. S., and M. Hayakawa (1992), Magnetospheric chorus emissions-A review, Planet. Space Sci., 40, 681-697.

Sergeev, V., A. Yakhnin, and N. Dmitrieva (1979), Substorms in the polar cap-Effect of high-velocity solar wind streams, Geomagn. Aeron., 19, $1121-1122$

Sibeck, D. G., R. W. McEntire, A. T. Y. Lui, R. E. Lopez, and S. M. Krimigis (1987), Magnetic field drift shell splitting: Cause of unusual dayside particle pitch angle distributions during storms and substorms, J. Geophys. Res., 92, 13,485-13,497.

Spanswick, E., E. Donovan, R. Friedel, and A. Korth (2007), Ground based identification of dispersionless electron injections, Geophys. Res. Lett., 34, L03101, doi:10.1029/2006GL028329.

Takahashi, K., B. J. Anderson, S.-i. Ohtani, G. D. Reeves, S. Takahashi, T. E. Sarris, and K. Mursula (1997), Drift-shell splitting of energetic ions injected at pseudo-substorm onsets, J. Geophys. Res., 102, 22,11722,130 .

Thorne, R. M., S. R. Church, W. J. Malloy, and B. T. Tsurutani (1977), The local time variation of ELF emissions during periods of substorm activity, J. Geophys. Res., 82, 1585-1590.

Tsurutani, B. T., and E. J. Smith (1974), Postmidnight chorus: A substorm phenomenon, J. Geophys. Res., 79, 118-127.

Tsurutani, B. T., and E. J. Smith (1977), Two types of magnetospheric ELF chorus and their substorm dependences, J. Geophys. Res., 82, 5112 5128

Tsyganenko, N. A., and D. P. Stern (1996), Modeling the global magnetic field of the large-scale Birkeland current systems, J. Geophys. Res., 101, 27, $187-27,198$

Waters, C. L., J. C. Samson, and E. F. Donovan (1995), The temporal variation of the frequency of high latitude field line resonances, J. Geophys. Res., 100, 7987-7996.

Weatherwax, A. T., T. J. Rosenberg, C. G. Maclennan, and J. H. Doolittle (1997), Substorm precipitation in the polar cap and associated Pc 5 modulation, Geophys. Res. Lett., 24, 579-582. 
Zaharia, S., C. Z. Cheng, and J. R. Johnson (2000), Particle transport and energization associated with substorms, J. Geophys. Res., 105, 18,74118,752 .

Zaharia, S., J. Birn, R. H. W. Friedel, G. D. Reeves, M. F. Thomsen, and C. Z. Cheng (2004), Substorm injection modeling with nondipolar, time-dependent background field, J. Geophys. Res., 109, A10211, doi:10.1029/2004JA010464.

R. Clauer, Virginia Institute of Technology, Blacksburg, VA 24061, USA D. Detrick and T. J. Rosenberg, Institute for Physical Science and Technology, University of Maryland, College Park, MD 20742, USA.

M. J. Engebretson, Department of Physics, Augsburg College, 2211 Riverside Avenue, Minneapolis, MN 55454, USA.

H. U. Frey and S. B. Mende, Space Sciences Laboratory, University of California, 7 Gauss Way, Berkeley, CA 94720, USA.

A. Gerrard and L. Lanzerotti, New Jersey Institute of Technology, Newark, NJ 07102, USA.
U. S. Inan and M. Spasojevic, Space Telecommunications and Radioscience Laboratory, Stanford University, Packard Building, Room 355, 350 Serra Mall, Palo Alto, CA 94305, USA.

J. LaBelle, Department of Physics, Dartmouth College, HB 6127 , Hanover, NH 03755, USA.

M. R. Lessard, Space Science Center, University of New Hampshire, 417 Morse Hall, Durham, NH 03824, USA. (marc.lessard@unh.edu)

N. J. Petit, Department of Computer Science, Augsburg College, Minneapolis, MN 55454, USA.

V. A. Pilipenko, Institute of the Physics of the Earth, Moscow, 117997, Russia.

A. Ridley, Center for Space Environment Modeling, University of Michigan, 1416 Space Research Building, 2455 Hayward Street, Ann Arbor, MI 48109, USA.

A. T. Weatherwax, Department of Physics, Siena College, Loudonville, NY 12211, USA. 